Textures and Microstructures, 1987, Vol. 7, pp. 85-93

Photocopying permitted by license only

(C) Gordon and Breach Science Publishers Inc., 1987

Printed in the United Kingdom

\title{
Orientations of the Cubic Unit Cell and Tetrahedron
}

\author{
J. C. CASATO $\dagger$ and W. G. FRICKE, Jr
}

$\dagger$ Aluminium Company of America, ALCOA Technical Centre PA 15069, USA

(Received 24 February 1986)

Charts are presented showing the cubic unit cell and tetrahedron for various simple orientations relative to the rolling plane and direction.

\section{INTRODUCTION}

The human mind is such that it doesn't truly appreciate threedimensional subjects until it perceives an image of a solid object well known to it. Generally, this means seeing a two-dimensional projection as though the three-dimensional object was viewed from a particular angle.

Crystallographic orientation, especially that of textures, is best appreciated by visualizing the crystallographic unit cell lying in relation to the specimen reference frame of sheet surface and rolling direction.

To make this visualization easier, perspective drawings have been made of the cube unit cell for various simple orientations that might be encountered in textures. Once the components of a texture are known for a particular specimen, certain of these drawings can be used to get a mental picture of the orientation of the grains in question. If several specimens are used to study the evolution of texture, a series of selected drawings can show how the grains rotate in the process. The unit cells can be annotated with numbers showing the strength of that component of a texture or the size of the cube can be varied to show the relative amount of this 
component. The object is to give the mind a picture of what is happening.

Because octahedral [111] planes are often used to determine grain orientation, it is often necessary to visualize the unit tetrahedron within the cubic cell. It suggests where the [111] poles for a particular grain would fall in the pole figure, for example. Moreover, the [111] faces of the tetrahedron are usually the potential slip planes and its edges are usually the slip directions, so in deformation studies it is important to visualize the tetrahedron.

Drawings of the cubic unit cell and tetrahedron were made for simple orientations.

\section{METHOD AND RESULTS}

A commercial plotting program $\dagger$ was used to make the drawings. Initially, to view the unit cube as it rotates to put various simple planes parallel to the plane of the drawing (the surface of a sheet specimen in a pole figure) angles were calculated so the view of the cube was in the direction of the poles of those simple, low index planes (Miller indices up to three). The rotated cubes were drawn in the positions those planes have in the basic stereographic triangle (Figure 1). Since these are perspective drawings, the back surface of the cubes appears to be smaller than the front surface. The same back face has also been cross-hatched in this figure to aid the visualization of the rotations involved.

Each small drawing has been labeled with the Miller indices of the crystallographic type plane that parallels the surface for that tilt. The tetrahedra defining the [111] type planes for the identical orientations are sketched in Figure 2.

So far the drawings have exhausted only two of the three degrees of freedom available to a solid that is orienting itself. The plane parallel to the surface has been defined, but the cube and tetrahedron are free to rotate around the pole perpendicular to that plane.

Larger drawings were made to define rotations in that plane for the cube (Figure 3 ) and the tetrahedron (Figure 4). Double-ended

† DISSPLA, software developed by ISSCO Graphics, 10505 Sorrento Valley Rd., San Diego, CA 92121. 


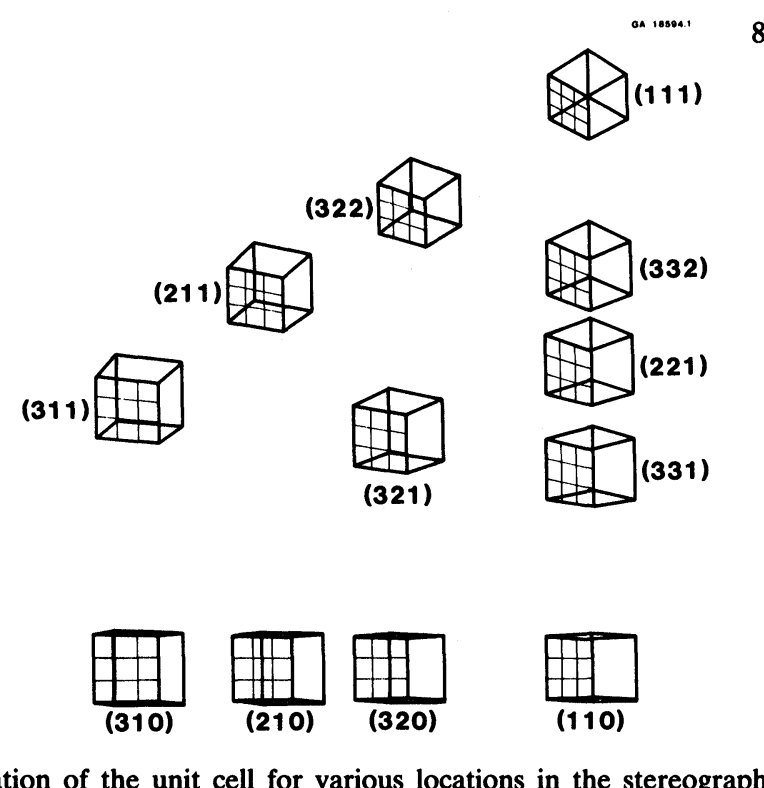

FIGURE 1 Orientation of the unit cell for various locations in the stereographic triangle.

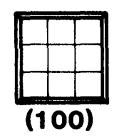

(1) 


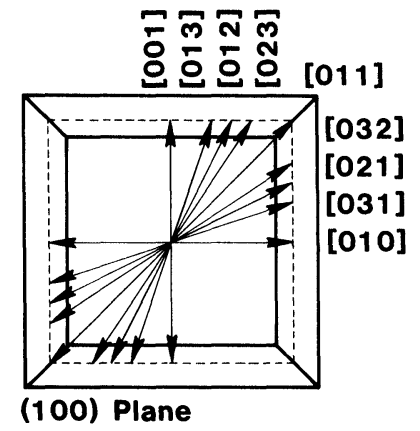

FIGURE 3 Directions in rolling plane for unit cell oriented in various ways.

GA 185044
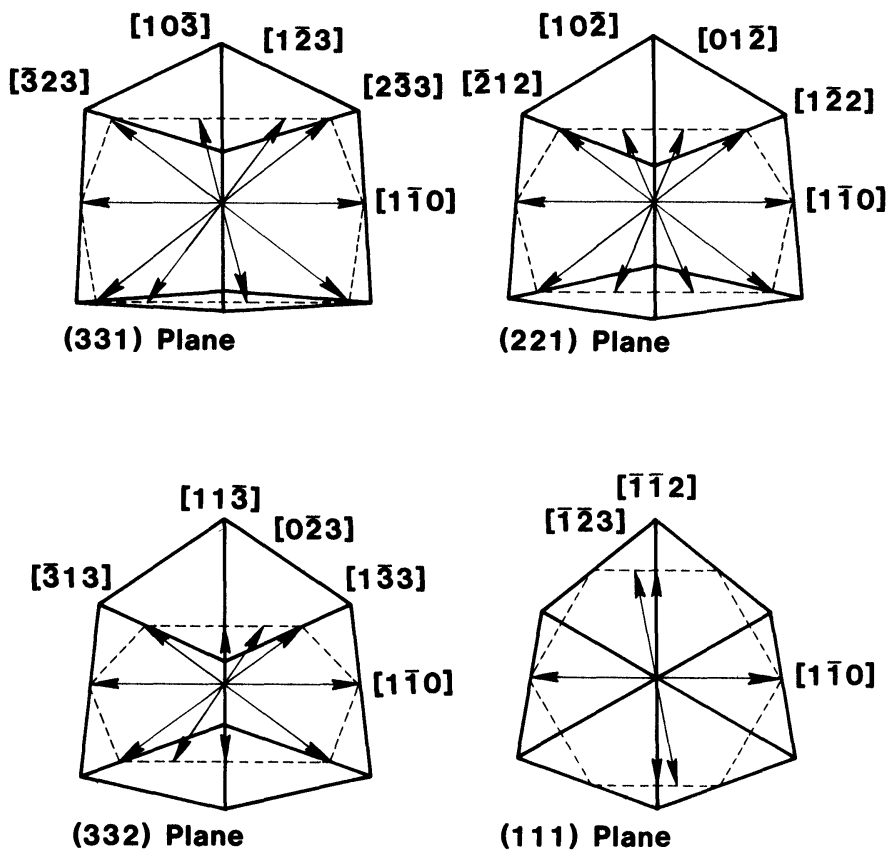

FIGURE 3 (Cont.) Directions in rolling plane for unit cell oriented in various ways. 

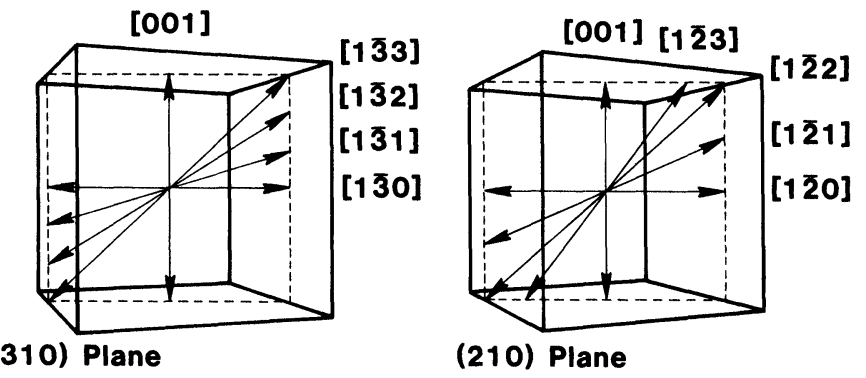

(210) Plane
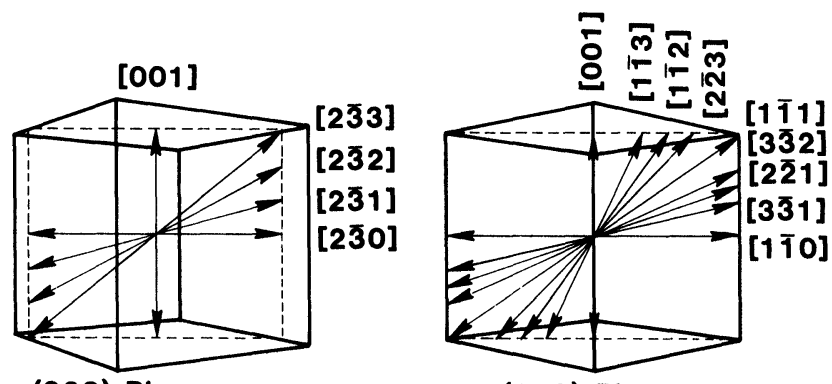

(110) Plane

FIGURE 3 (Cont.) Directions in rolling plane for unit cell oriented in various ways.

arrows were put inside the drawing of the three-dimensional object to define simple directions that lie in the surface plane. All of the arrows go through the center of the solid and extend to the surface of the unit cube. They all lie in the plane of the drawing. To aid visualization of the plane of the drawing (which is also the crystallographic plane noted), a dashed line is drawn showing where the plane intersects the cube (not the tetrahedron). Half of the solid lies above the drawing and half lies below.

Each arrow is marked with crystallographic indices. The numbers represent type only because reversing directions along the line changes the signs of the indices. In some cases, for clarity, not every variant of a type is shown. 

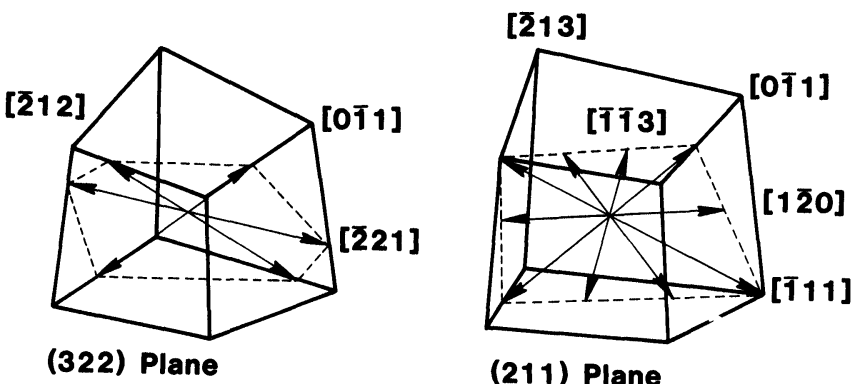

(211) Plane

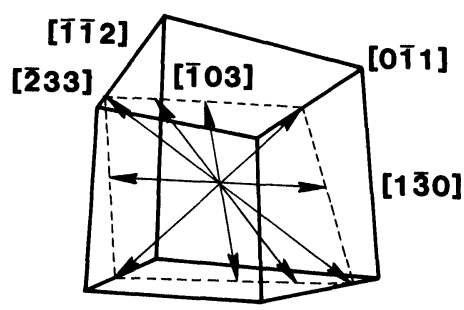

(311) Plane

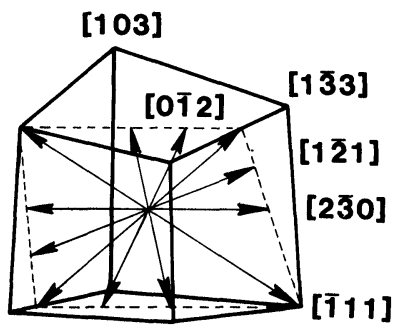

(321) Plane

FIGURE 3 (Cont.) Directions in rolling plane for unit cell oriented in various ways. Note: All vectors lie in plane of paper and reach the surface of the unit cube. Dashed line shows where indicated plane (the rolling plane), which passes through the center of the cube, intersects the surfaces of the cube.

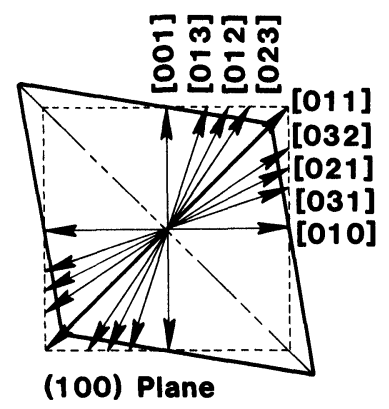

FIGURE 4 Directions in rolling plane for tetrahedron oriented in various ways. 


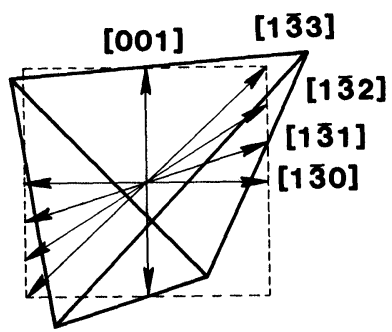

(310) Plane

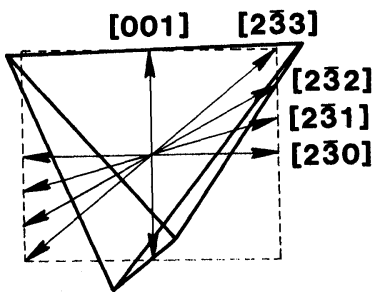

(320) Plane

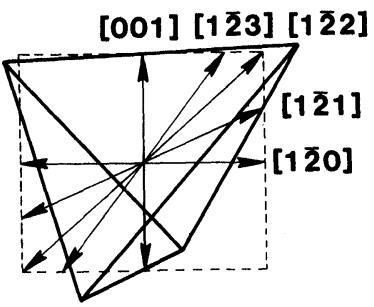

(210) Plane

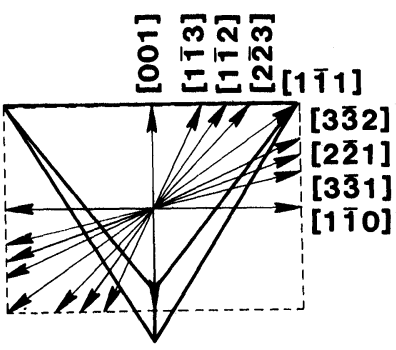

(110) Plane

FIGURE 4 (Cont.) Directions in rolling plane for tetrahedron oriented in various ways.

OA 18504.9

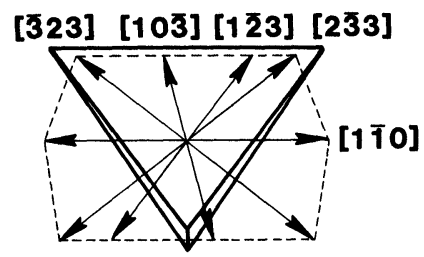

(331) Plane

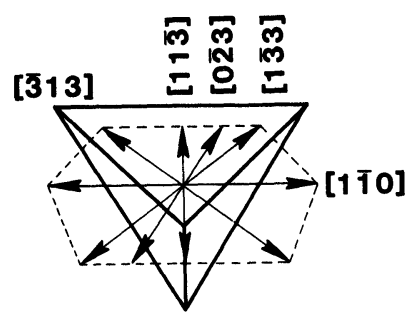

(332) Plane

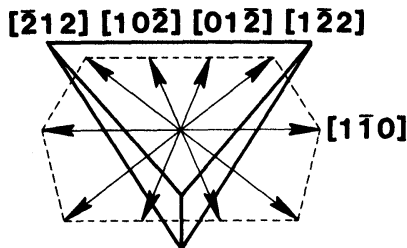

(221) Plane

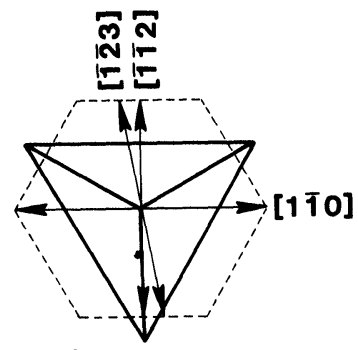

(111) Plane

FIGURE 4 (Cont.) Directions in rolling plane for tetrahedron oriented in various ways. 


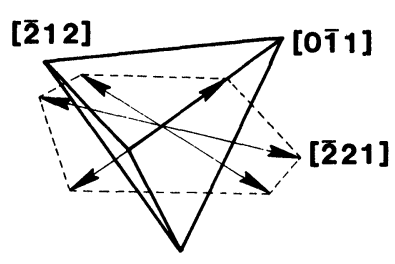

(322) Plane

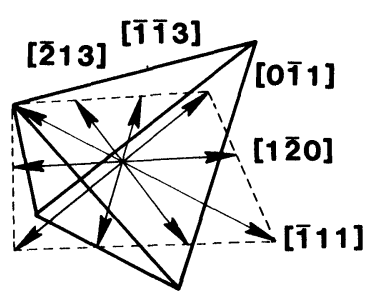

(211) Plane

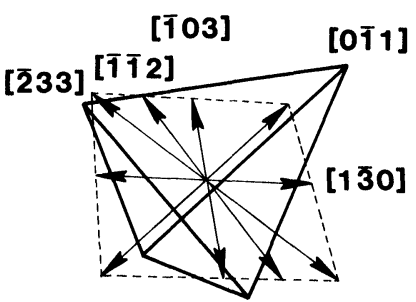

(311) Plane

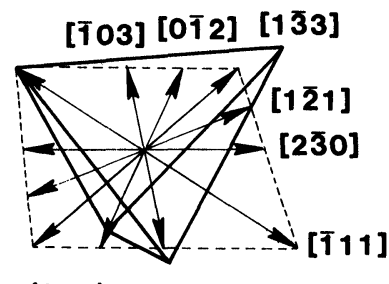

(321) Plane

FIGURE 4 (Cont.) Directions in rolling plane for tetrahedron oriented in various ways. Note: All vectors lie in plane of paper and reach the surface of the unit cube. Dashed line shows where indicated plane (the rolling plane), which passes through the center of the cube, intersects the surfaces of the cube.

\section{CONCLUSION}

For visualization, then, of the components of a crystallographic texture, when the notation is the metallurgical one of the plane parallel to the specimen surface and the direction in that plane pointing in the rolling direction, one would select the drawing of the cube appropriate to the plane and rotate it until the appropriate direction was vertical, such as the rolling direction is usually drawn in a pole figure. It is probably best to trace part of the drawing at this point to simplify it.

Alongside this drawing one could sketch other components of a mixed texture or the component present at a previous time in the life of the specimen, or the like. If a series of sketches is made, care 
should be taken that the cube makes an orderly progression from one drawing to the next because mirror images of these drawings are possible.

If deformation is part of the study, it is also useful to sketch the tetrahedron for the same orientations in order to visualize how the slip planes and directions lie in relation to the specimen surface and stress direction. 